\title{
Methionine as an antimutagen in Schizosaccharomyces pombe
}

\author{
By C. H. CLARKE* \\ M.R.C. Mutagenesis Research Unit, \\ Institute of Animal Genetics, Edinburgh, 9
}

(Received 7 October 1964)

\begin{abstract}
SUMMARY
Methionine inhibition of the phenotypic expression of $a d n^{-} \rightarrow a d n^{+}$ reverse mutations in the fission yeast Schizosaccharomyces pombe was investigated. This inhibition occurred solely with L-methionine, and not with a variety of other growth factors. The inhibitory effect on revertant expression did not apply to a number of reverse and forward mutations other than $a d n^{-} \rightarrow a d n^{+}$. Alteration of the adenine supply to $a d n^{-}$ organisms before and after mutagen treatment had no effect. Methionine was shown to have additional inhibitory effects on the leakiness of an $a d n^{-}$mutant, on complementation between $a d n-1$ mutants, and on the growth of certain $a d n^{-}$strains in the presence of the analogue 6-methylaminopurine. It is suggested that $\mathrm{S}$-adenosylmethionine may be implicated in these methionine effects.
\end{abstract}

\section{INTRODUCTION}

In earlier papers the presence of methionine in the minimal plating medium was shown to inhibit the phenotypic expression of newly arising reverse mutations to adenine-independence in Schizosaccharomyces pombe (Clarke, 1962, 1963). In doubly auxotrophic adenine- and methionine-dependent $\left(a d n^{-} m e t^{-}\right)$strains this methionine effect led to cases of an apparent influence of the methionine allele upon the reverse mutability of the $a d n^{-}$allele. In these earlier experiments methionine in the plating medium inhibited the phenotypic expression of revertants of spontaneous, nitrous acid-, and ultraviolet radiation-induced origin. There was no effect on the viability of $a d n^{-}$organisms which had been treated with a mutagen and then plated on a medium containing a high concentration of adenine ( $50 \mu \mathrm{g} . / \mathrm{ml}$.). Residual divisions of $a d n^{-}$organisms were decreased on a minimal medium supplemented with methionine. Methionine was without effect on adenine-independent $\left(a d n^{+}\right)$reversions once these had been phenotypically expressed.

The present paper describes the results of experiments designed to give a better understanding of the mode of action of methionine in Schizosaccharomyces pombe. In particular it was sought to learn whether methionine alone had this antimutagenic effect, or whether other growth factors acted similarly. Experiments were made to see whether there was a critical dependence upon the methionine concentration in the minimal medium, and whether the methionine effect was in reality merely a delay in the appearance of $a d n^{+}$revertants and which would not be

* Present address: Genetics Foundation, The University of Texas, Austin, Texas 78712, U.S.A. 
apparent if revertant colonies were scored later than the routine 7 -day period. The possibility that methionine might act only during a critical period before the actual phenotypic expression of $a d n^{+}$reversions was examined by experiments in which there was a delayed removal of methionine from populations of $\mathrm{HNO}_{2}$-treated organisms on minimal medium. Witkin (1956) and Clavilier, Luzzati \& Slonimski (1960) had been able to show by similar experiments that there were critical periods for the action of amino acids in ultraviolet radiation-mutagenesis in bacteria, and the histidine inhibition of revertants arising by a gene conversion process in the $a d_{3}$ system of Saccharomyces cerevisiae.

Since the earlier experiments had suggested that methionine inhibition of revertant expression might perhaps be the result of decreased residual divisions on minimal medium, attempts were made to test this. Conditions which were tested included alteration of the growth conditions for the organisms before mutagen treatment, starvation for adenine and other growth factors before mutagen treatment, and allowing limited supply or synthesis of adenine after treatment with the mutagen. Although, as mentioned, no influence of methionine had been found upon the viability of mutagen-treated organisms upon minimal medium with a high concentration of adenine ( $50 \mu \mathrm{g} . / \mathrm{ml}$.), further tests were made on medium containing only a low concentration of adenine. The specificity of the methionine effect was examined in a variety of reverse and forward mutation systems, as was also the influence of methionine upon other aspects of cell physiology: complementation of adenine mutants, leakiness, and growth on the purine analogue 6-methylaminopurine.

\section{METHODS}

Details of media, adenine-1 mutants of Schizosaccharomyces pombe used, and the routine procedures for nitrous acid and ultraviolet radiation reverse mutation experiments have all been given previously (Leupold, 1955; Clarke, 1962, 1963). The suspensions of organisms treated usually contained initially $2-5 \times 10^{7}$ viable organisms $/ \mathrm{ml}$. The source of ultraviolet radiation delivered about $90 \%$ of its ouput at $2537 \AA$; a dose rate of approximately $14.7 \mathrm{ergs} / \mathrm{mm}^{2} / \mathrm{sec}$. was used. Because of supply difficulties 'Oxoid' Agar No. 3 had to be used for some experiments in place of the usual 'Oxoid' Ionagar No. 2.

Abbreviations used: $a d n^{-}=$adenine-requiring; $a d n^{+}=$adenine-independent; Adn = adenine, routinely used at $50 \mu \mathrm{g} . / \mathrm{ml}$; Met $=$ L-methionine, routinely used at $40 \mu \mathrm{g} . / \mathrm{ml}$.; MMA = minimal medium agar; YEA = yeast extract agar; GSVB $=$ Giese's salts vitamins buffer; $h^{+}$and $h^{-}$are the two heterothallic mating-types of Schizosaccharomyces pombe.

\section{RESULTS}

The earlier experiments revealed the effects of L-methionine on the phenotypic expression of $a d n^{+}$reversions, on the residual divisions of $a d n^{-}$organisms on adenine-deficient medium, on the differential loss of viability of $a d n^{-}$organisms on minimal medium with and without methionine, and on the result of delayed addition of methionine to minimal medium plates bearing mutagen-treated adnorganisms.

The unique activity of L-methionine. In the earlier experiments the methionine 
had been sterilized, often in minimal medium, by autoclaving (121 ${ }^{\circ}, 16 \mathrm{~min}$.). Experiments in which filter-sterilized methionine was added to MMA plates showed that a product formed from methionine during autoclaving was not responsible for the inhibitory action on revertant expression. Experiments in which filtersterilized L-, DL-, and D-methionine, DL-homocysteine, L-cysteine, DL-cystathionine, mercaptoethylamine (cysteamine) and choline were tested separately showed that the inhibition of $a d n^{+}$mutation expression was obtained uniquely with L-methionine. DL-methionine (at $80 \mu \mathrm{g} . / \mathrm{ml}$.) had the same action as L-methionine at $40 \mu \mathrm{g} . / \mathrm{ml}$. Experiments in which the pools of amino acids, purines and pyrimidines used by Lederberg (1950) were added to MMA gave no indication that any other of these growth factors could, at comparable concentrations, mimic the inhibitory action of L-methionine on $a d n^{+}$revertant expression.

The influence of $\mathrm{L}-$ methionine concentration on the inhibition of revertant expression. Figure 1 shows the result of experiments in which nitrous acid-treated organisms of mutant $a d n-1,25$ were plated on MMA containing graded concentrations of L-methionine in the range $1 \cdot 25-80 \mu \mathrm{g} . / \mathrm{ml}$. Concentrations of $20 \mu \mathrm{g} . / \mathrm{ml}$. and above exerted maximal inhibitory activity on $a d n^{+}$revertant expression; concentrations of 1.25 and $2.5 \mu \mathrm{g} . / \mathrm{ml}$. gave no definite inhibition; concentrations between 5 and $20 \mu \mathrm{g} . / \mathrm{ml}$. gave intermediate degrees of inhibition.

The time course of appearance of $a d n^{+}$revertant colonies in the presence and absence of L-methionine. $A d n^{+}$revertant colonies were scored routinely after incubation for 7 days at $30^{\circ}$. Figure 2 shows the time course of appearance of $a d n^{+}$revertants of mutant $a d n-1,25$ induced by $\mathrm{HNO}_{2}$ treatment, on minimal medium with and without methionine. On minimal medium most of the $a d n^{+}$revertants formed colonies which could be scored after 7 days at $30^{\circ}$, and only a few additional revertants appeared when incubation was extended to 15 days. In the experiment shown in Fig. 2, no further $a d n^{+}$colonies appeared on MMA during the second week of incubation.

On minimal medium + L-methionine (40 $\mu \mathrm{g} . / \mathrm{ml}$.), however, there was a slow continual rise in the number of revertant colonies which appeared on the plates over the 2-week period. Incubation of the plates for longer periods led to the appearance of very many minute colonies, some of which were revertants with an extremely slow growth rate, and others of which resulted from $a d n^{-}$organisms able to grow into minute colonies on the adenine released by dead organisms or $a d n^{+}$revertant colonies.

The transient nature of methionine inhibitory activity. Experiments were made in which $a d n$ - mutants were treated with $\mathrm{HNO}_{2}$ and then spread on cellophan disks placed on the surface of methionine-supplemented minimal medium plates. In this way it was possible to study the effect of temporary incubation in the presence of methionine, followed by continued incubation on methionine-free minimal medium. Control experiments with a methionine auxotroph showed that there was no detectable carry-over of methionine by the cellophan disks. Figure 3 shows the results of two such delayed methionine removal experiments, with $\mathrm{HNO}_{2}$-treated mutant $a d n-1,25$. There was a very slow loss of potential revertants, expressed on subsequent transfer and incubation on minimal medium, over extended periods of incubation in the presence of methionine. This was most probably a consequence of the loss of viability of $a d n^{-}$organisms on methionine-supplemented minimal 
medium (Clarke, 1963). Otherwise potential $a d n^{+}$revertants were not permanently inhibited in their subsequent phenotypic expression by transient exposure to methionine.

The influence of pre-treatment growth conditions. Organisms for $a d n^{-} \rightarrow a d n^{+}$ reverse mutation experiments were grown routinely in aerated liquid yeast extract medium + adenine ( $\mathrm{YE}+\mathrm{Adn})$. It was thus of interest to see whether methionine inhibition of $a d n^{+}$phenotypic expression could be modified or abolished by growing $a d n^{-}$organisms under different conditions before mutagen treatment. Mutant $a d n-1,25$ was grown in liquid aerated media of the following types: minimal +

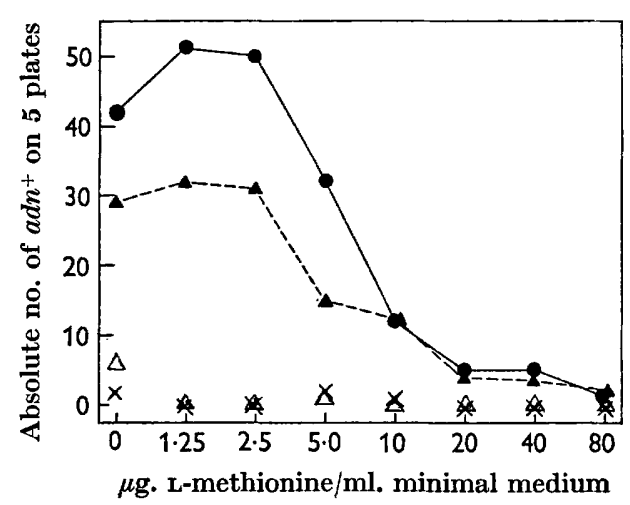

Fig. 1

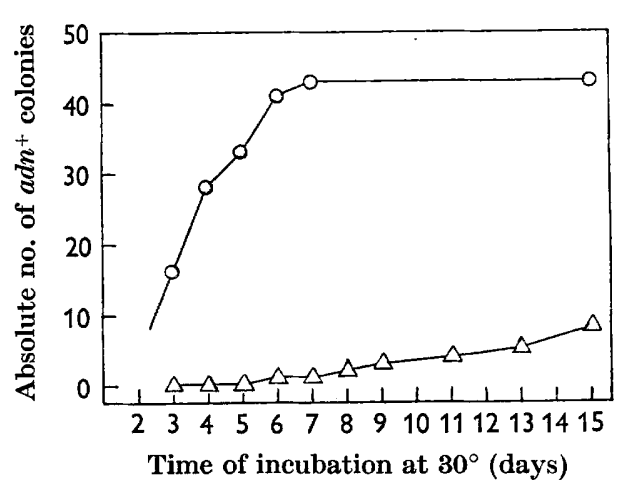

Fig. 2

Fig. 1. The influence of $L$-methionine concentration on inhibition of phenotypic expression of $\mathrm{HNO}_{\mathrm{z}}$-induced revertants of adn-1, 25 mutant of Schizosaccharomyces pombe. Experiment A: $x=$ untreated controls; $O=\mathrm{HNO}_{2}$-treated samples (76.6\% survival). Experiment $B: \Delta=$ controls; $\Delta=\mathrm{HNO}_{2}$-treated series (95\% survival).

Fig. 2. Time course of appearance of revertant colonies of $\mathrm{HNO}_{2}$-treated $a d n-1,25$ mutant of Schizosaccharomyces pombe (90.5\% survival). $O=$ minimal medium; $\Delta=$ minimal $+\mathrm{L}$-methionine $(40 \mu \mathrm{g}$. $/ \mathrm{ml}$.).

adenine, minimal + hypoxanthine, and minimal + inosinic acid (di-sodium salt). In all cases methionine inhibition of $a d n^{+}$expression occurred, regardless of how the $a d n^{-}$organisms had been grown before nitrous acid treatment.

The influence of pre-treatment starvation. Adenine-1 mutants 25 and 51 were grown as usual in liquid aerated YE + Adn medium, harvested by centrifugation and incubated for $24 \mathrm{hr}$ at $30^{\circ}$ in aerated liquid media deficient in a nitrogen source $\left(0.1 \%, \mathrm{w} / \mathrm{v}, \mathrm{KH}_{2} \mathrm{PO}_{4}\right.$, glucose, vitamins, adenine), or deficient in adenine but containing amino acids (minimal medium + casein hydrolysate), or deficient in adenine (minimal medium). Organisms pre-incubated under these three sets of conditions were then tested for methionine inhibition of the expression of $a d n^{+}$revertants induced by $\mathrm{HNO}_{2}$ treatment. In no case was there found an abolition or obvious diminution of the methionine effect. However, $a d n^{-}$organisms pre-incubated in liquid minimal medium appeared to be much more sensitive to the lethal action of nitrous acid than did organisms not so pre-starved.

The influence of trace adenine on the methionine effect. Samples of agars from different sources contain different concentrations of contaminating adenine (Dr M. Luzzati, personal communication), and thus allow different degrees of residual 
growth of $a d n^{-}$organisms when used for the preparation of minimal medium. Experiments were made in which $a d n^{-}$organisms were treated with $\mathrm{HNO}_{2}$, then plated for $a d n^{+}$reversions on minimal medium and on minimal medium + methionine, and containing $1.6 \%(\mathrm{w} / \mathrm{v})$ of different specimens of agar. Those used were 'Difco' Purified Agar, 'Difco' Bacto Agar, 'Oxoid' Ionagar No. 2, and 'Oxoid' Agar No. 3. The first-named of these agars contains very little adenine indeed (Dr M. Luzzati, personal communication) while the last-named gives a final concentration of the order of $0 \cdot 1 \mu \mathrm{g}$. adenine $/ \mathrm{ml}$. when used in the preparation of minimal medium. The use of these different agars did not affect the degree of

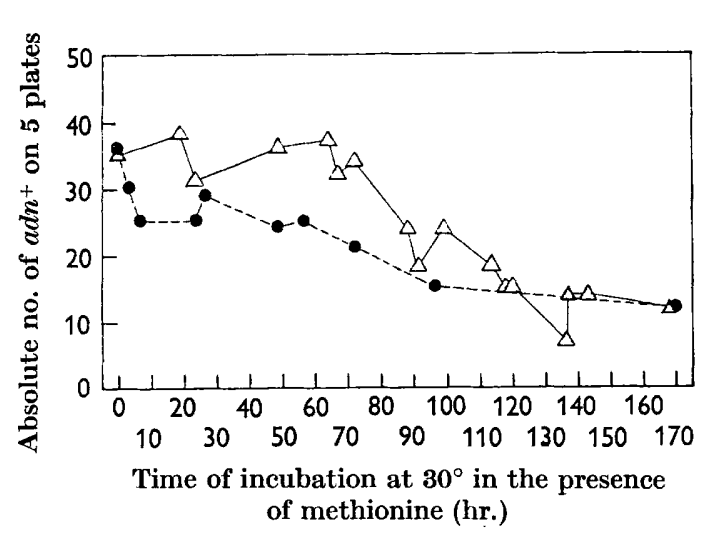

Fig. 3

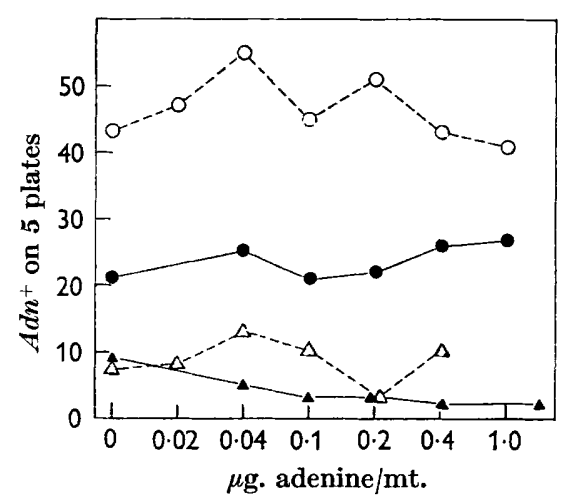

Fig. 4

Fig. 3. Delayed methionine removal experiments with $\mathrm{HNO}_{2}$-treated adn-1, 25 mutant of Schizosaccharomyces pombe. $\triangle=$ experiment $\mathrm{A} ; \boldsymbol{O}=$ experiment $\mathrm{B}$.

Fig. 4. The influence of low concentration adenine supplementation of minimal medium \pm methionine on the expression of $\mathrm{HNO}_{2}$-induced adn-1 revertants of Schizosaccharomyces pombe. $\bigcirc=$ minimal medium agar (MMA), mutant $a d n-1,25 ; 0=$ MMA, mutant adn1, 51; $\triangle=$ MMA + methionine (Met), mutant adn-1, 25; $\Delta=$ MMA + Met, mutant $a d n-1,51$. Mutant $a d n-1,25,32 \cdot 6 \%$ survival; mutant $a d n-1,51,21 \cdot 1 \%$ survival.

methionine inhibition detectably, even when pre-starvation for adenine (see above) was combined with plating on minimal medium, with and without methionine, containing the very pure 'Difco' Purified Agar.

Figure 4 shows the influence of low degrees of adenine supplementation of minimal medium, with and without methionine, upon the yield of $a d n^{+}$revertants obtained on treating mutants $a d n-1,25$ and $a d n-1,51$ with $\mathrm{HNO}_{2}$. Within the range used $(0 \cdot 02-1 \cdot 0 \mu \mathrm{g}$. adenine $/ \mathrm{ml}$.) there was no noticeable influence of trace adenine upon the expression of $\mathrm{HNO}_{2}$-induced revertants. It is not possible to work much beyond the upper value of adenine supplementation used here because of the increasingly heavy background growth of the unreverted $a d n^{-}$organisms.

Temporary incubation on high concentration adenine medium. Experiments were made in which $a d n$ - organisms, after treatment with $\mathrm{HNO}_{2}$, were spread on cellophan disks and incubated on minimal medium + adenine, at $50 \mu \mathrm{g} . / \mathrm{ml}$. After known periods of incubation sets of membranes were transferred to minimal medium or minimal medium + methionine plates. The usable period of incubation on adenine was limited by the development of heavy background growth of the $a d n^{-}$organisms. In all experiments the methionine effect on revertant expression was not abolished 
or obviously decreased by periods of post-treatment incubation on high-concentration adenine medium up to $10 \mathrm{hr}$ at $30^{\circ}$.

Use of a temperature-sensitive auxotroph. Mutant adn-1, 199 is temperature sensitive, being $a d n^{-}$at $30^{\circ}$ but $a d n^{+}$at $25^{\circ}$. Organisms of this mutant were treated with $\mathrm{HNO}_{2}$ or ultraviolet radiation and then incubated on minimal medium at $25^{\circ}$ for limited periods. In this way it was hoped to provide conditions for temporary intracellular production of adenine, rather than mere extracellular supply as in the experiments described above. The effect of this temporary incubation at $25^{\circ}$ was tested upon subsequent expression of $a d n^{+}$reversions expressed at $30^{\circ}$ in the absence and presence of methionine. Methionine inhibition, weak in the case of $\mathrm{HNO}_{2}$-induced $a d n^{+}$, strong for ultraviolet-induced revertants, was not abolished by incubation up to $11 \mathrm{hr}$ at $25^{\circ}$.

Viable count assays on low-concentration adenine medium. It had been shown earlier (Clarke, 1963) that addition of methionine did not affect estimates of viable counts made with untreated or mutagen-treated organisms when these were plated on minimal media supplemented with adenine $50 \mu \mathrm{g} . / \mathrm{ml}$. Because low degrees of adenine supplementation did not abolish the effect of methionine on $a d n^{+}$expression, it was decided to test the effect of methionine upon viability as assayed at low concentrations of adenine. Organisms of an $a d n^{-}$mutant were treated with $\mathrm{HNO}_{2}$ and colony counts made on minimal medium + adenine $0.5 \mu \mathrm{g} . / \mathrm{ml}$., in the presence and absence of methionine. The colonies, which were very small, were scored after 7 days at $30^{\circ}$; the counts were the same on both media. These counts were of necessity made at much lower plating densities than was used for estimates of $a d n^{+}$revertants. On the reversion plates the amount of adenine available for each $a d n^{-}$organism was less by a factor of about $10^{5}$ than was the case on the viability plates.

Growth rate of adn + revertants. Samples of $10^{-5}$ dilution of a culture of two $\mathrm{HNO}_{2}$ induced revertants of mutant $a d n-1,51$ were sprayed at daily intervals on the surface of minimal medium, and minimal medium + methionine plates, bearing a background of $\mathrm{HNO}_{2}$-treated $a d n-1,51$. The added $a d n^{+}$organisms formed colonies, on the methionine-free and the methionine-containing minimal medium, which were identical in size after 7 days at $30^{\circ}$. Earlier examination of the plates showed that colonies formed by the added $a d n^{+}$organisms reached full size about 1 day earlier on minimal medium than on minimal medium + methionine. Thus there was a weak inhibition of the growth of $a d n^{+}$organisms but this was certainly not the explanation for the methionine effect on revertant expression.

Influence of methionine on other reverse mutations. In addition to the mutants for which methionine inhibition of revertant expression has been reported earlier (Clarke, 1963), Table 1 and Table 2 show other reverse mutation systems which were tested. Methionine inhibition seems to be general for $a d n^{-} \rightarrow a d n^{+}$reversions, and there seems to be an effect on at least those $\mathrm{HNO}_{2}$-induced revertants of leucine auxotroph leu-3, 241 which formed scorable colonies after incubation for 7 days. Methionine had no obvious inhibitory effect on the expression of $\mathrm{HNO}_{2}$-induced reversions of the following arginine and lysine auxotrophs: arg-D 14, arg-G 2, lys-3 I, lys-7 A, lys-4B, lys-6 B, and lys-6, 2. The results with di-auxotrophic adn1, 25.lys-7 $\mathrm{A}$ and $a d n-1,25 . \arg -\mathrm{G} 2$ strains show that methionine inhibition was definitely restricted to the $a d n^{+}$reversions. 


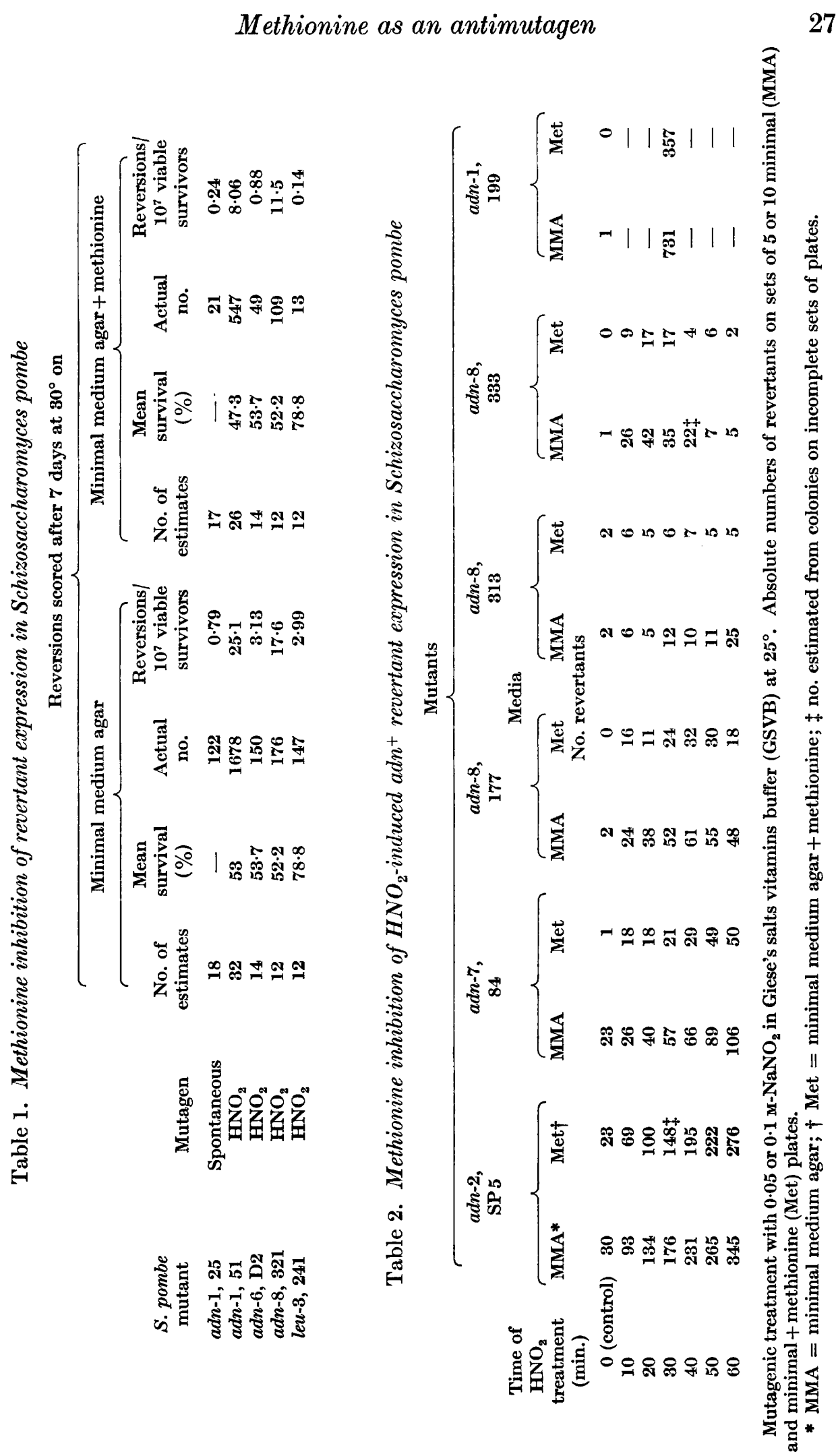


Forward mutation systems. Nitrous acid-induced mutations of the wild-type of Schizosaccharomyces pombe from sensitivity to resistance to 5-fluorouracil $50 \mu \mathrm{g} . / \mathrm{ml}$. were not affected by the addition of methionine to the minimal medium +5 -fluorouracil upon which these mutations were scored. Forward mutations at the $a d n-1$, $-3,-4,-5$ and perhaps also other loci (Heslot, 1960; Clarke \& Anwar Nasim, 1964) can be scored as white colonies or sectors among the red colonies of an unmutated $a d n-7$ strain, on a medium containing limiting adenine. Mutant $a d n-7,407$, which has a very low spontaneous and $\mathrm{HNO}_{2}$-induced rate of reversion to $a d n^{+}$, was treated with $\mathrm{HNO}_{2}$ and plated on minimal medium containing adenine at $20 \mu \mathrm{g} . / \mathrm{ml}$., with or without methionine. The results obtained indicated no inhibition of the expression of induced $a d n^{-}$forward mutations, though methionine did intensify the red colour of the $a d n-7$ colonies.

Leakiness. Mutant $a d n-7,486$ is leaky when incubated on minimal medium at $30^{\circ}$, so that a heavy film of background growth is formed. Supplementation of the minimal medium with methionine resulted in a drastic inhibition of this background, as shown in Pl. 1, fig. 1.

6-Methylaminopurine. Certain double adenine mutants of the type $a d n-7, a d n-1$, $-3,-4,-5$ or -9 can grow slowly on 6 -methylaminopurine as an alternative to adenine though the $a d n-7$ mutant alone cannot do so ( $\mathrm{Dr}$ H. Heslot, personal communication). Plating a large number of $a d n-7$ organisms on minimal medium containing 6-methylaminopurine thus selected at least some of the spontaneous double $a d n-7, a d n^{-}$mutants present in the population. Minute white, pink and red colonies appeared after 10-15 days of incubation at $30^{\circ}$. Addition of methionine to the medium prevented the appearance of most of these colonies, and the growth of most isolated $a d n-7, a d n^{-}$strains on 6-methylaminopurine. This effect is shown in Pl. 1, fig. 2.

Complementation. Plating of large numbers of ascospores from a cross of the type $a d n-1, a, h^{+} \times a d n-1, h h^{-}$, between two non-identical $a d n-1$ mutants which complement, results in the growth on minimal medium of $a d n^{+}$colonies which are of two types. There are $a d n^{+}$wild-type recombinants (Leupold, 1957) and also complementing diploids and aneuploids, many of which are unstable and undergo meiosis when heterozygous for mating-type (Professor U. Leupold, personal communication). Plating on minimal medium + methionine can, at least in some cases, result in a marked inhibition of the growth of the $a d n^{+}$complementing colonies, though not of the $a d n^{+}$recombinants. This effect is shown in Pl. 2.

Reverse mutations in bacteria. Salmonella typhimurium auxotroph ath-A2 (Yura, 1956) was derived, by transduction with phage PLT 22 grown on wild-type bacteria, from the di-auxotrophic met-F 31, ath-A2 (kindly supplied by Dr S. W. Glover). Mutant ath-A 2 has a simultaneous requirement for adenine and thiamine. Ultraviolet irradiation of this mutant induces $a t h^{+}$revertants (Yura, 1956). In the present experiments $\boldsymbol{S}$. typhimurium ath-A 2 organisms, grown in aerated nutrient broth, were irradiated in saline with doses of ultraviolet radiation of about 140, 290, and $540 \mathrm{ergs} / \mathrm{mm}^{2}$, and plated for ath $^{+}$revertants. Four media were used: minimal medium A (Lederberg, 1950) with and without methionine, and with and without a supplement of $1 \%(\mathrm{v} / \mathrm{v})$ nutrient broth. $A t h^{+}$revertants were scored after 3 days at $30^{\circ}$. There was a very strong effect of the broth supplement in increasing the revertant yield (Witkin, 1956), but no methionine inhibition was seen. 


\section{DISCUSSION}

The action of L-methionine in inhibiting the phenotypic expression of newly arising $a d n^{+}$reverse mutations in Schizosaccharomyces pombe appears to be restricted to $\mathrm{L}$-methionine, and mainly to $a d n^{+}$reversions. An apparent inhibition of $\mathrm{HNO}_{2}$ induced reversions of the leucine auxotroph leu-3, 241 requires explanation and further study. It is possible that there is an effect of methionine upon the viability of $l e u^{-}$organisms which have been treated with nitrous acid; this inhibition applies only to those $l e u^{+}$reversions scored after 7-day incubation. Many of the induced $l e u^{+}$reversions appeared later on the minimal agar plates (up to 2 weeks of incubation) and were most probably of a suppressor type, like the slowly growing revertants studied in the $S$. pombe arg-1, 230 system by Heslot (1962). The leu- mutant has a very high leucine requirement for full growth on minimal medium, and until the biochemical genetics of the $l e u^{+}$revertants are studied it would be premature to suggest possible mechanisms by which methionine could inhibit leu $u^{+}$expression or growth rate.

That methionine has inhibitory activity on leakiness and complementation of adenine auxotrophs and on their growth on a purine analogue indicates that methionine probably affects a biochemical step common to several metabolic processes. An underlying and unifying biochemical mechanism for the varied effects of methionine described here and previously (Clarke, 1963) may lie in the formation of S-adenosylmethionine (Gawel, Turner \& Parks, 1962). In a turnip slice system Dr D. D. Davies (1964, and personal communication) found that methionine inhibited respiration, and that its action was annulled by adenine. Formation of S-adenosylmethionine, from ATP and methionine, which can accumulate in the vacuoles of plant and yeast cells, might well lead to a low availability of ADP and ATP in the cell. In this way a 'resting cell' condition might be produced, in which such processes as synthesis of new enzymes in newly mutated or complementing diploid cells or residual cell divisions would not occur. S-adenosylmethionine does not accumulate in bacteria (Gawel et al. 1962) so that the absence of an inhibitory effect on $a t h^{+}$reversions in Salmonella typhimurium might be expected. One would expect the inhibitory activities of L-methionine in Schizosaccharomyces pombe to be counteracted by high concentrations of adenine, but this is, of course, impossible to test in an $a d n^{-} \rightarrow a d n^{+}$reverse mutation system.

The experiments described in this paper show that the methionine inhibition of $a d n^{+}$expression in Schizosaccharomyces pombe is not readily modified by a variety of adenine removal or addition treatments applied before or after mutagen treatment. The experiments in which methionine was removed from populations of $\mathrm{HNO}_{2}$-treated organisms incubated on minimal medium showed that there was no critical period for methionine action. Potential $a d n^{+}$revertants remained expressible on subsequent incubation in the absence of methionine, even after quite long periods on methionine-containing medium. High concentrations of methionine, of the order of $400 \mu \mathrm{g} . / \mathrm{ml}$., have been shown by Dr M. Luzzati (personal communication) to simulate the effect of low concentrations of histidine in inhibiting the appearance of $a d n^{+} \mathrm{his}^{+}$revertants, formed by a gene conversion process, in heteroallelic diploid adn-3 (adn-his $\left.{ }^{-}\right)$strains of Saccharomyces cerevisiae (Clavilier et al. 1960). It has been shown in the present work that methionine inhibition of $a d n^{+}$ 
revertant expression in $S$. pombe is not restricted either to those reversions due to repair at the original adenine locus, or to suppressor mutations. Both types of revertants occur (Clarke, unpublished results) and methionine inhibits the phenotypic expression of each. It is possible that adenine might under certain conditions inhibit the expression of met $^{+}$reversions. No evidence has been found for this up till now, in adn-met- diauxotrophic strains, but the use of a monoauxotrophic met ${ }^{-}$strain which is completely blocked, together with tests of revertants induced by a variety of mutagens, would allow this question to be investigated.

I am grateful to Dr Charlotte Auerbach, F.R.S., for her interest, enthusiasm and encouragement, to Professor U. Leupold and Dr H. Heslot for details of unpublished results and for strains of Schizosaccharomyces pombe, and to Drs B. J. Kilbey, M. Luzzati, N. Loprieno, G. Kølmark and J. Cummins for interesting and useful discussions. I wish to thank Dr D. D. Davies particularly for his suggestions on the possible role of S-adenosylmethionine in the $S$. pombe phenomena. I am grateful also to Roche Products Ltd for the supply of 5-fluorouracil.

\section{REFERENCES}

Clarke, C. H. (1962). A case of mutagen specificity attributable to a plating medium effect. Z. Vererb.-Lehre, 93, 435.

Clarke, C. H. (1963). Suppression by methionine of reversions to adenine independence in Schizosaccharomyces pombe. J. gen. Microbiol. 31, 353.

Clarke, C. H. \& Anwar Nasim, M. (1964). Mutant expression delay in a nitrous acid forward mutation system in Schizosaccharomyces pombe. (Abstract). Heredity, 19, 530.

Cluavilier, L., Luzzati, M. \& Slonimski, P. P. (1960). Sur la conversion du gène ad3 chez la levure. C. r. Séanc. Soc. Biol. 154, 1970.

Davies, D. D. (1964). The effect of methionine on the metabolism of disks of storage tissue. J. exp. Bot. 15, 538.

Gawel, L. J., Turner, J. R. \& Parks, L. W. (1962). Accumulation of S-adenosylmethionine by microorganisms. J. Bact. 83, 497.

Heslot, H. (1960). Schizosaccharomyces pombe: un nouvel organisme pour l'étude de la mutagenèse chimique. Abh. dt. Akad. Wiss. Berl., Klasse Medizin J., p. 98.

Heslot, H. (1962). Étude quantitative de réversions biochimiques induites chez la levure Schizosaccharomyces pombe par des radiations et des substances radiomimétiques. $A b h$. dtsch. Akad. Wiss. Berl., Klasse Medizin J. 1962, p. 193.

LEDERBERG, J. (1950). Isolation and characterization of biochemical mutants of bacteria. Meth. med. Res. 3, 5.

LEUPOLD, U. (1955). Versuche zur genetischen Klassifizierung adeninabhängiger Mutanten von Schizosaccharomyces pombe. Arch. Klaus-Stift. VererbForsch. 30, 506.

LEUPoLd, U. (1957). Physiologisch-genetische Studien an adeninabhängiger Mutanten von Schizosaccharomyces pombe. Schreiz. Z. Path. Bakt. $20,535$.

WITKIN, E. M. (1956). Time, temperature and protein synthesis: a study of ultravioletinduced mutation in bacteria. Cold. Spr. Harb. Symp. quant. Biol. 21, 123.

YURA, T. (1956). Evidence of non-identical alleles in purine-requiring mutants of Salmonella typhimurium. Genetic Studies with Bacteria. Carneg. Inst. Wash. Publ. 612, 63. 
Journal of General Microbiology, Vol. 39, No. 1

Plate 1

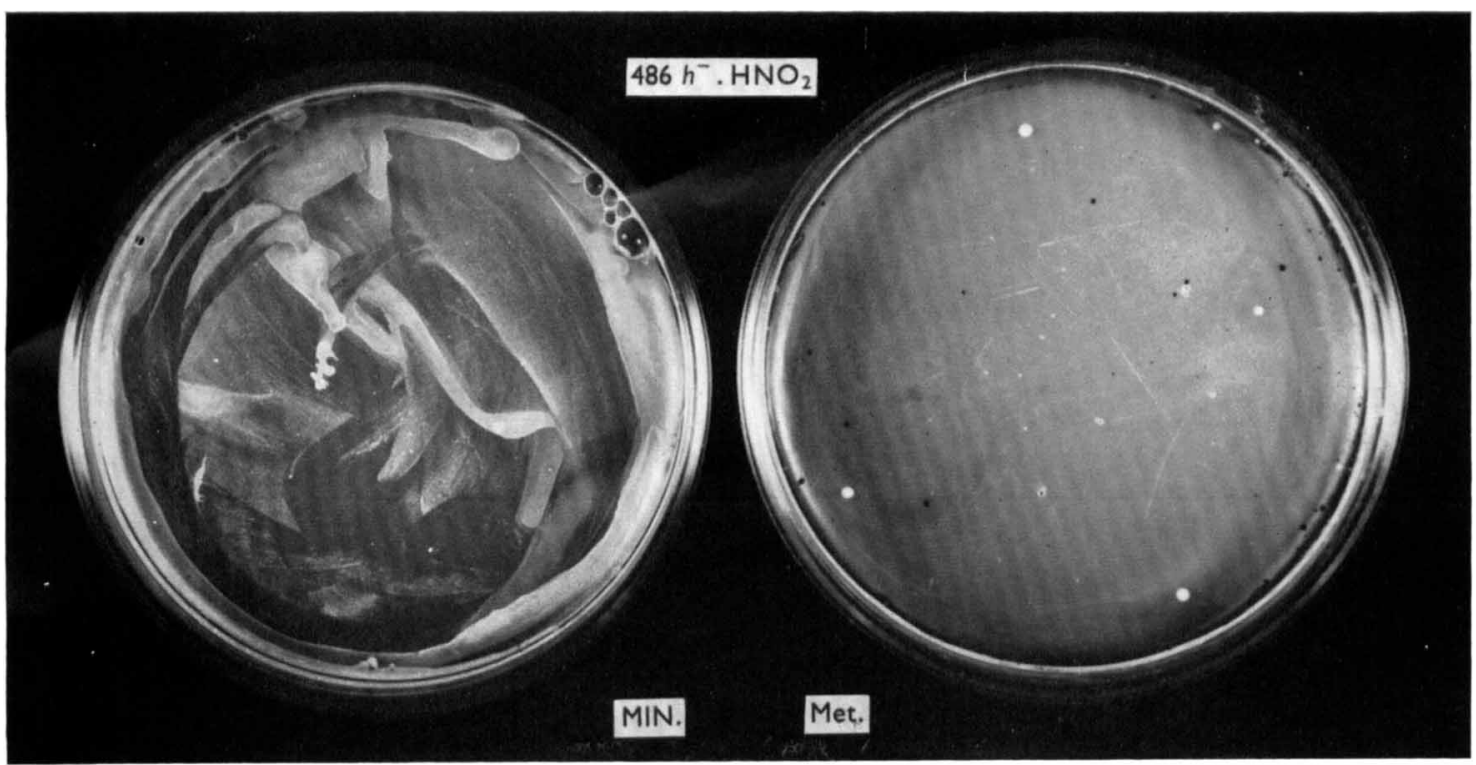

Fig. 1

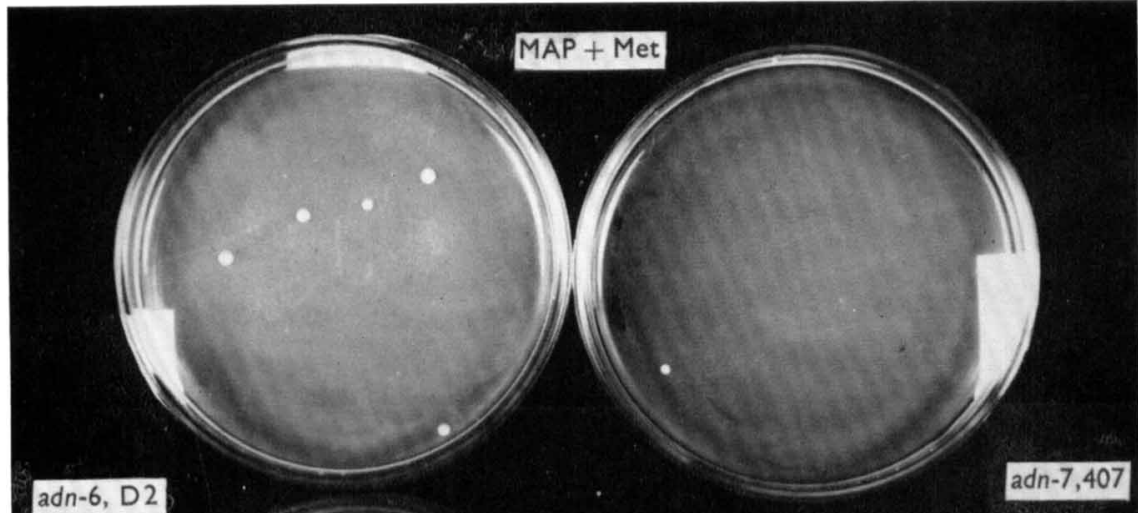

adn-6, D2

adn-7,407

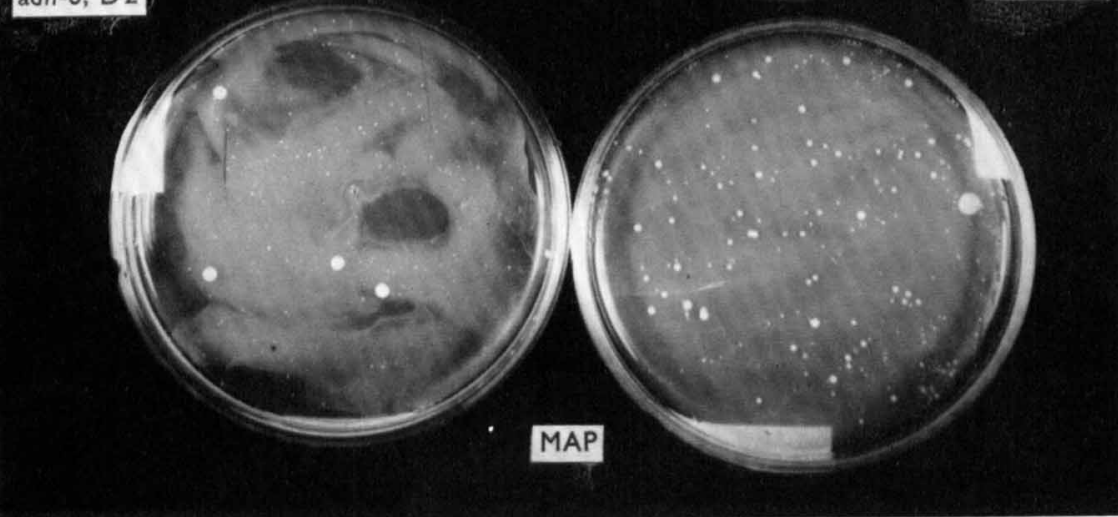

Fig. 2

C. H. CLARKE

(Facing p. 30) 
Journal of General Microbiology, Vol. 39, No. 1

Plate 2

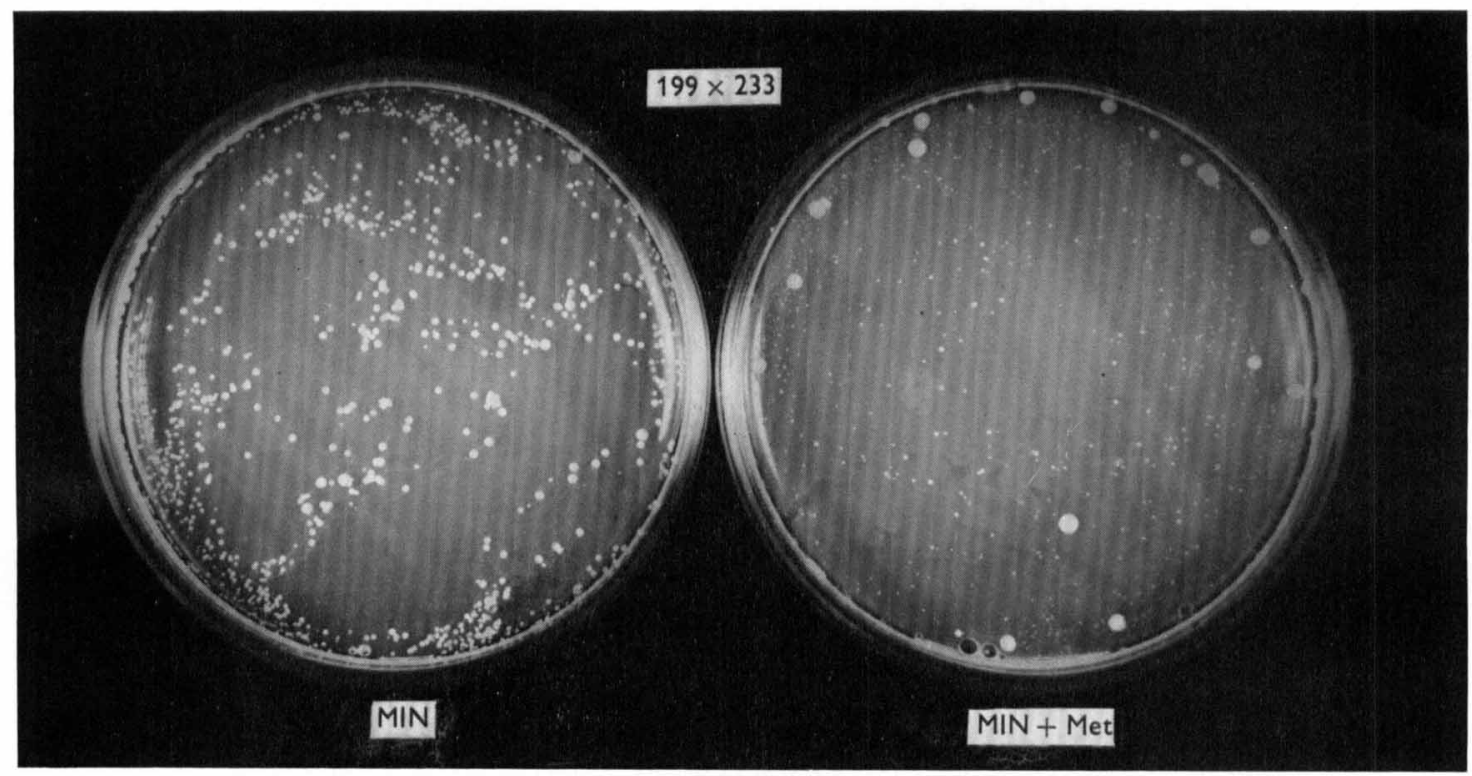

C. H. CLARKE 


\section{EXPLANATION OF PLATES}

\section{Plate 1}

Fig. 1. Residual growth of $\mathrm{HNO}_{2}$-treated organisms of Schizosaccharomyces pombe mutant $a d n-7,486 h^{-}$on minimal medium (MIN) and minimal + methionine (Met). Heavy film of residual growth on the minimal medium; very light background growth with several $a d n^{+}$revertant colonies on the minimal + methionine medium.

Fig. 2. Methionine inhibition of the growth of adenine double mutants of $S$. pombe on 6-methylaminopurine. The two plates on the left have been spread with organisms of mutant adn-6, D2; those on the right with mutant $a d n-7,407$. The two lower plates contain minimal medium + 6-methylaminopurine (MAP); the upper two plates minimal medium +6-methylaminopurine + methionine (MAP + Met). On the MAP plates colonies of $a d n^{+}$revertants and smaller colonies of $a d n^{-} a d n^{-}$double mutants are visible; on the MAP + Met plates only $a d n^{+}$revertant colonies are visible.

\section{Prate 2}

Methionine inhibition of complementation between two adn-1 mutants of $S$. pombe. Large numbers of ascospores from the cross $a d n-1,199 \times a d n-1,233$ were plated on minimal medium (MIN) and on minimal + methionine (MIN + Met). On the minimal medium colonies of $a d n^{+}$recombinants and of complementing diploids and aneuploids cannot be distinguished by size. On the minimal medium + methionine $a d n^{+}$recombinants form larger colonies; the complementing diploids and aneuploids form small colonies. 